\title{
On the heat-flow distribution from and the temperature profile on an equally heated rectangle, calculated by series development
}

Citation for published version (APA):

Nieuwenhuizen, J. K. (1985). On the heat-flow distribution from and the temperature profile on an equally heated rectangle, calculated by series development. International Journal for Numerical Methods in Engineering, 21(12), 2257-2272. https://doi.org/10.1002/nme.1620211210

DOI:

10.1002/nme.1620211210

Document status and date:

Published: 01/01/1985

Document Version:

Publisher's PDF, also known as Version of Record (includes final page, issue and volume numbers)

Please check the document version of this publication:

- A submitted manuscript is the version of the article upon submission and before peer-review. There can be important differences between the submitted version and the official published version of record. People interested in the research are advised to contact the author for the final version of the publication, or visit the $\mathrm{DOI}$ to the publisher's website.

- The final author version and the galley proof are versions of the publication after peer review.

- The final published version features the final layout of the paper including the volume, issue and page numbers.

Link to publication

\footnotetext{
General rights

- You may freely distribute the URL identifying the publication in the public portal. follow below link for the End User Agreement:

www.tue.nl/taverne

Take down policy

If you believe that this document breaches copyright please contact us at:

openaccess@tue.nl

providing details and we will investigate your claim.
}

Copyright and moral rights for the publications made accessible in the public portal are retained by the authors and/or other copyright owners and it is a condition of accessing publications that users recognise and abide by the legal requirements associated with these rights.

- Users may download and print one copy of any publication from the public portal for the purpose of private study or research.

- You may not further distribute the material or use it for any profit-making activity or commercial gain

If the publication is distributed under the terms of Article $25 \mathrm{fa}$ of the Dutch Copyright Act, indicated by the "Taverne" license above, please 


\title{
ON THE HEAT-FLOW DISTRIBUTION FROM AND THE TEMPERATURE PROFILE ON AN EQUALLY HEATED RECTANGLE, CALCULATED BY SERIES DEVELOPMENT
}

\author{
J. K. NIEUWENHUIZEN \\ Technische Hogeschool, Eindhoven, The Netherlands
}

\begin{abstract}
SUMMARY
A method is proposed to approximate the solutions of a certain class of differential equations, linear or nonlinear, in two or three dimensions, provided that the boundary conditions are given on a rectangle or a parallelepiped, respectively. For other boundary shapes the co-ordinate system must be transformed to meet that requirement. As in illustration, an example is given for the solution of Poisson's equation in two dimensions with a constant heat source, giving the temperatures on the rectangle together with the heat-flow distribution along its edges. The basis of the method is a Taylor-series development around one point; the result is given in terms of as many partial derivatives in that point as is desired. A similar method has already been described by Small $^{2}$ for the 'heat equation' $\partial \theta / \partial t=\partial^{2} \theta / \partial x^{2}$. Compared with finite difference and finite element solutions, these methods have the advantage that the solution is continuous, whereas first and second derivatives such as heat fluxes are available at hardly any effort. The results are compared with those of the exact solution. Even if the size of the determinant is limited to $4 \times 4$, the accuracy is already better than 98.98 per cent. When more effort is spent to solve a system of 10 equations, the accuracy is better than 99.95 per cent.
\end{abstract}

\section{METHOD}

Consider a function $\theta_{[x, y, z]}$ which is continuous in all its derivatives. Then

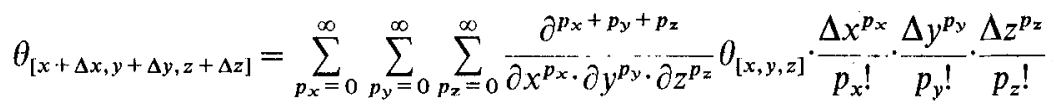

Writing for short

$$
\left\{p_{x}, p_{y}, p_{z}, \theta\right\} \text { for } \frac{\partial^{p_{x}+p_{y}+p_{z}}}{\partial x^{p_{x}} \cdot \partial y^{p_{y}} \cdot \partial z^{p_{z}}} \theta_{[x, y, z]}
$$

one has

$$
\theta_{[x+\Delta x, y+\Delta y, z+\Delta z]}=\sum_{p_{x}=0}^{\infty} \sum_{p_{y}=0}^{\infty} \sum_{p_{z}=0}^{\infty}\left\{p_{x}, p_{y}, p_{z}, \theta\right\} \cdot \frac{\Delta x^{p_{x}}}{p_{x} !} \cdot \frac{\Delta y^{p_{x}}}{p_{y} !} \cdot \frac{\Delta z^{p_{z}}}{p_{z} !}
$$

If the boundaries coincide with planes for $x, y, z$ is constant respectively, they put relatively simple restrictions on the possible functions $\left\{p_{x}, p_{y}, p_{n}, \ldots\right\}$; the differential equation $L(\theta, x, y, z)=0$ can be worked out to relations between those functions.

As soon as sufficient equations are developed for the desired number of functions, they may-- under the usual conditions - be solved. As soon as $\left\{p_{x}, p_{y}, p_{z}, \theta\right\}$ are known for all $p_{x}+p_{y}+p_{z}<r$, the value of $\theta$ at any $x, y, z$ can be approximated by a Taylor series.

As a simple example, we consider a rectangle which extends from $x=-1$ to $x=+1$ and 
from $y=-b$ to $y=+b$. Poisson's equation reads

where

$$
\frac{\partial^{2} \theta}{\partial x^{2}}+\frac{\partial^{2} \theta}{\partial y^{2}}=f_{[x, y]} \quad\left[\frac{K}{m^{2}}\right]
$$

$$
f_{[x, y]}=-\frac{1}{\lambda} \cdot q
$$

or

$$
\frac{1}{l^{2}} \cdot \frac{\partial^{2} \theta}{\partial \xi^{2}}+\frac{1}{b^{2}} \cdot \frac{\partial^{2} \theta}{\partial \eta^{2}}=f_{[\xi, \eta]} ; \quad \xi:=\frac{x}{1}, \eta:=\frac{y}{b}
$$

whereas the boundary conditions are

Consequently

$$
\theta=\theta_{r} \text { for } \xi= \pm 1 \text { and for } \eta= \pm 1
$$

$$
\begin{aligned}
\sum_{p_{\xi}=0}^{\infty} \sum_{p_{\eta}=0}^{\infty} \frac{1}{l^{2}} \cdot\left\{p_{\xi}+2, p_{\eta}, \theta\right\} & \cdot \frac{\xi^{p_{\xi}}}{p_{\xi} !} \cdot \frac{\eta^{p_{\eta}}}{p_{\eta} !}+\sum_{p_{\xi}=0}^{\infty} \sum_{p_{\eta}=0}^{\infty} \frac{1}{b^{2}} \cdot\left\{p_{\xi}, p_{\eta}+2, \theta\right\} \cdot \frac{\xi^{p_{\xi}}}{p_{\xi} !} \cdot \frac{\eta^{p_{\eta}}}{p_{\eta} !} \\
= & \sum_{p_{\xi}=0}^{\infty} \sum_{p_{\eta}=0}^{\infty}\left\{p_{\xi}, p_{\eta}, f\right\} \cdot \frac{\xi^{p_{\xi}}}{p_{\xi} !} \cdot \frac{\eta^{p_{\eta}}}{p_{\eta} !}
\end{aligned}
$$

Since this should be valid for all $-1<=\xi<=+1$ and $-1<=\eta<=+1$, Poisson's equation is equivalent to

$$
\left.\left.\frac{1}{l^{2}} \cdot\left\{p_{\xi}+2, p_{\eta}, \theta\right\}+\frac{1}{b^{2}} \cdot\left\{p_{\xi}, p_{\eta}+2, \theta\right\}=\left\{p_{\xi}, p_{\eta}, f\right\} ; p_{\xi}, p_{\eta}\right\rangle=0 \quad \text { while } p_{\xi}, p_{\eta}\right\rangle=0
$$

\section{THE BOUNDARY CONDITIONS}

Since $\theta_{[1, \eta]}$ is available for $-1<=\eta\left\langle=+1\left\langle 0, p_{\eta}, \theta\right\rangle_{[1,0]}\right.$ is known for every $\left.p_{\eta}\right\rangle=0$. Together with similar conditions at $[-1,0],[0,1]$ and $[0,-1]$, boundary conditions of type 1 (i.e. $\theta$ given on boundary) lead to

$$
\begin{aligned}
\sum_{m=0}^{\infty} \frac{1}{(2 m) !}\{2 m, 2 n, \theta\} & =\theta_{r} \text { for } n=0 ; \\
& =0 \text { for } n\langle>0 ; \\
\sum_{m=0}^{\infty} \frac{1}{(2 m) !}\{2 m, 2 n+1, \theta\} & =0 ; \\
\sum_{m=0}^{\infty} \frac{1}{(2 m+1) !}\{2 m+1, n, \theta\} & =0 ;
\end{aligned}
$$

Similarly for $n$ :

$$
\begin{aligned}
\sum_{n=0}^{\infty} \frac{1}{(2 n) !}\{2 m, 2 n, \theta\} & =\theta_{r} \text { for } m=0 \\
& =0 \text { for } m<>0 \\
\sum_{n=0}^{\infty} \frac{1}{(2 n) !}\{2 m+1,2 n, \theta\} & =0 ; \\
\sum_{n=0}^{\infty} \frac{1}{(2 n+1) !}\{m, 2 n+1, \theta\} & =0
\end{aligned}
$$


whereas Poisson reads

$$
\frac{1}{l^{2}} \cdot\{m+2, n, \theta\}+\frac{1}{b^{2}} \cdot\{m, n+2, \theta\}=\{m, n, f\}
$$

or, with $c:=b / 1$

$$
\{m, n+2, \theta\}=b^{2} \cdot\{m, n, f\}-c^{2} \cdot\{m+2, n, \theta\}
$$

is $\{m, n, f\}=0$ for $m>0$ or $n>0$ and then $n=0$, odd series:

$$
\frac{1}{1 !}\{1,0,0\}+\frac{1}{3 !}\{3,0,0\}+\frac{1}{5 !}\{5,0, \theta\}+\frac{1}{7 !}\{7,0, \theta\}+\cdots=0
$$

$n=2$, odd serics:

or

$$
\frac{1}{1 !}\{1,2, \theta\}+\frac{1}{3 !}\{3,2, \theta\}+\frac{1}{5 !}\{5,2, \theta\}+\frac{1}{7 !}\{7,2,0\}+\cdots=0
$$

$$
-\frac{c^{2}}{1 !}\{3,0,0\}-\frac{c^{2}}{3 !}\{5,0,0\}-\frac{c^{2}}{5 !}\{7,0, \theta\}+\cdots=0
$$

so that

$$
\begin{array}{r}
\frac{1}{1 !}\{1,0, \theta\}+\frac{1}{3 !}\{3,0, \theta\}+\frac{1}{5 !}\{5,0, \theta\}+\frac{1}{7 !}\{7,0, \theta\}+\cdots=0 \\
\frac{1}{1 !}\{3,0, \theta\}+\frac{1}{3 !}\{5,0, \theta\}+\frac{1}{5 !}\{7,0, \theta\}+\cdots=0 \\
\frac{1}{1 !}\{5,0, \theta\}+\frac{1}{3 !}\{7,0, \theta\}+\cdots=0
\end{array}
$$

from which $\{2 m+1, n, \theta\}=0$

$$
\{m, 2 n+1, \theta\}=0
$$

and also $\quad\{2 m+1,2 n+1, \theta\}=0$

$n=0$, even series:

$$
\frac{1}{0 !}\{0,0, \theta\}+\frac{1}{2 !}\{2,0, \theta\}+\frac{1}{4 !}\{4,0, \theta\}+\frac{1}{6 !}\{6,0,0\}+\cdots=\theta_{r}
$$

$m=0$, even series

or

$$
\frac{1}{0 !}\{0,0, \theta\}+\frac{1}{2 !}\{0,2, \theta\}+\frac{1}{4 !}\{0,4, \theta\}+\frac{1}{6 !}\{0,6, \theta\}+\cdots=0_{r}
$$

$$
\frac{1}{0 !}\{0,0, \theta\}-\frac{c^{2}}{2 !}\{2,0, \theta\}+\frac{c^{4}}{4 !}\{4,0, \theta\}-\frac{c^{6}}{6 !}\{6,0, \theta\}+\cdots=0_{r}-\frac{b^{2}}{2 !} \cdot\{0,0, f\}
$$

$n=2$, even series:

$$
\frac{1}{0 !}\{0,2, \theta\}+\frac{1}{2 !}\{2,2, \theta\}+\frac{1}{4 !}\{4,2, \theta\}+\frac{1}{6 !}\{6,2, \theta\}+\cdots=0
$$


or

$$
\begin{gathered}
-c^{2} \cdot \frac{1}{0 !}\{2,0, \theta\}-\frac{c^{2}}{2 !}\{4,0, \theta\}-\frac{c^{2}}{4 !}\{6,0, \theta\}-\frac{c^{2}}{6 !}\{8,0, \theta\}+\cdots=-b^{2}\{0,0, f) \\
\frac{1}{0 !}\{2,0, \theta\}+\frac{1}{2 !}\{4,0, \theta\}+\frac{1}{4 !}\{6,0,0\}+\frac{1}{6 !}\{8,0, \theta\}+\cdots=l^{2}\{0,0, f\}
\end{gathered}
$$

$m=2$, even series:

$$
\frac{1}{0 !}\{2,0, \theta\}+\frac{1}{2 !}\{2,2, \theta\}+\frac{1}{4 !}\{2,4, \theta\}+\frac{1}{6 !}\{2,6, \theta\}+\cdots=0
$$

or

$$
\frac{1}{0 !}\{2,0, \theta\}-\frac{c^{2}}{2 !}\{4,0, \theta\}+\frac{c^{4}}{4 !}\{6,0,0\}-\frac{c^{6}}{6 !}\{6,0, \theta\}+\cdots=0
$$

This gives the following table of coefficients for

$$
\{0,0, \theta\}-\theta_{r},\{2,0, \theta\},\{4,0, \theta\},\{6,0, \theta\}, \ldots,\{18,0, \theta\}:
$$

$\frac{1}{0 !} \quad \frac{1}{2 !} \quad \frac{1}{4 !} \quad \frac{1}{6 !} \quad \frac{1}{8 !} \quad \frac{1}{10 !} \quad \frac{1}{12 !} \quad \frac{1}{14 !} \quad \frac{1}{16 !} \quad \frac{1}{18 !} \quad 0$

$\frac{1}{0 !}-\frac{c^{2}}{2 !}+\frac{c^{4}}{4 !}-\frac{c^{6}}{6 !}+\frac{c^{8}}{8 !}-\frac{c^{10}}{10 !}+\frac{c^{12}}{12 !}-\frac{c^{14}}{14 !}+\frac{c^{16}}{16 !}-\frac{c^{18}}{18 !}-\frac{c^{2}}{2 !} \cdot b l\{0,0, f\}$

$\frac{1}{0 !} \quad \frac{1}{2 !} \quad \frac{1}{4 !} \quad \frac{1}{6 !} \quad \frac{1}{8 !} \quad \frac{1}{10 !} \quad \frac{1}{12 !} \quad \frac{1}{14 !} \quad \frac{1}{16 !}+\frac{1}{c} \cdot b l\{0,0, f\}$

$$
\frac{1}{0 !}-\frac{c^{2}}{2 !}+\frac{c^{4}}{4 !}-\frac{c^{6}}{6 !}+\frac{c^{8}}{8 !}-\frac{c^{10}}{10 !}+\frac{c^{12}}{12 !}-\frac{c^{14}}{14 !}+\frac{c^{16}}{16 !} \quad 0
$$

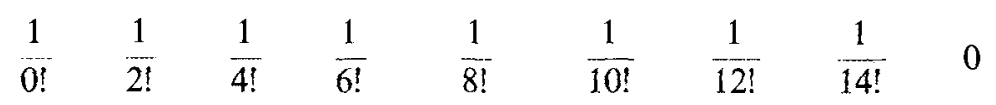

$\frac{1}{0 !}-\frac{c^{2}}{2 !}+\frac{c^{4}}{4 !}-\frac{c^{6}}{6 !}+\frac{c^{8}}{8 !}-\frac{c^{10}}{10 !}+\frac{c^{12}}{12 !}-\frac{c^{14}}{14 !} \quad 0$

$\begin{array}{cccccccc}1 & 1 & 1 & 1 & 1 & \frac{1}{10 !} & \frac{1}{12 !} & 0\end{array}$

$\frac{1}{0 !}-\frac{c^{2}}{2 !}+\frac{c^{4}}{4 !}-\frac{c^{6}}{6 !}+\frac{c^{8}}{8 !}-\frac{c^{10}}{10 !}+\frac{c^{12}}{12 !} \quad 0$

$\frac{1}{0 !} \frac{1}{2 !} \quad \frac{1}{4 !} \quad \frac{1}{6 !} \quad \frac{1}{8 !} \quad \frac{1}{10 !} \quad 0$

$\frac{1}{0 !}-\frac{c^{2}}{2 !}+\frac{c^{4}}{4 !}-\frac{c^{6}}{6 !}+\frac{c^{8}}{8 !}-\frac{c^{10}}{10 !} \quad 0$ 
In a first manual approximation, we restrict ourselves to the four unknowns $\{0,0, \theta\}-\theta_{r}$, $\{2,0, \theta\},\{4,0, \theta\},\{6,0, \theta\}$ :

$$
\begin{array}{ccccc}
\frac{1}{0 !} & \frac{1}{2 !} & \frac{1}{4 !} & \frac{1}{6 !} & 0 \\
\frac{1}{0 !} & -\frac{c^{2}}{2 !} & +\frac{c^{4}}{4 !} & -\frac{c^{6}}{6 !} & -\frac{c^{2}}{2 !} \cdot b l\{0,0, f\} \\
& \frac{1}{0 !} & \frac{1}{2 !} & \frac{1}{4 !} & \frac{1}{c} \cdot b l\{0,0, f\} \\
1 & -\frac{c^{2}}{2 !} & +\frac{c^{4}}{4 !} & 0
\end{array}
$$

With

$$
\operatorname{Det}=\frac{1}{6 ! \cdot 2 !} \cdot\left(\frac{1}{c}+c\right) \cdot\left(\frac{1}{c^{3}}+c^{3}\right)-\frac{1}{4 ! \cdot 4 !} \cdot\left(\frac{1}{c^{2}}-c^{2}\right)^{2}-\frac{1}{4 ! \cdot 2 ! \cdot 2 !} \cdot\left(\frac{1}{c}+c\right)^{2}
$$

One finds

$$
\begin{gathered}
\{0,0, \theta\}-\theta_{r}=\frac{b l\{0,0, f\}}{\operatorname{Det}} *\left[\left(\frac{1}{c^{3}}+c^{3}\right) \cdot\left(\frac{1}{4 ! \cdot 4 ! \cdot 2 !}-\frac{1}{6 ! \cdot 2 ! \cdot 2 !}\right)+\left(\frac{1}{c}+c\right) \cdot\left(\frac{1}{4 ! \cdot 2 ! \cdot 2 ! \cdot 2 !}-\frac{1}{6 ! \cdot 4 !}\right)\right. \\
\{2,0, \theta\}=\frac{b l\{0,0, f\}}{\operatorname{Det}} *\left[\left(\frac{1}{c^{3}}+c^{3}\right) \cdot \frac{1}{6 ! \cdot 2 !}+\left(\frac{1}{c^{2}}-c^{2}\right) \cdot \frac{c}{4 ! \cdot 4 !}-\left(\frac{1}{c}+c\right) \cdot \frac{1}{4 ! \cdot 2 ! \cdot 2 !}\right. \\
\{0,2, \theta\}=\frac{b l\{0,0, f\}}{\operatorname{Det}} *\left[\left(\frac{1}{c^{3}}+c^{3}\right) \cdot \frac{1}{6 ! \cdot 2 !}-\left(\frac{1}{c^{2}}-c^{2}\right) \cdot \frac{1}{4 ! \cdot 4 ! \cdot c}-\left(\frac{1}{c}+c\right) \cdot \frac{1}{4 ! \cdot 2 ! \cdot 2 !}\right] \\
\{4,0, \theta\}=\frac{b l\{0,0, f\}}{\operatorname{Det}} \cdot \frac{1}{c^{2}} *\left[\left(\frac{1}{c^{3}}+c^{3}\right) \cdot \frac{1}{6 !}-\left(\frac{1}{c}+c\right) \cdot \frac{1}{4 ! \cdot 2 !}\right] \\
\{2,2, \theta\}=\frac{b l(0,0, f\}}{\operatorname{Det}} *\left[-\left(\frac{1}{c^{3}}+c^{3}\right) \cdot \frac{1}{6 !}+\left(\frac{1}{c}+c\right) \cdot \frac{1}{4 ! \cdot 2 !}\right] \\
\{0,4, \theta\}=\frac{b l\{0,0, f\}}{\operatorname{Det}} \cdot c^{2} *\left[\left(\frac{1}{c^{3}}+c^{3}\right) \cdot \frac{1}{6 !}-\left(\frac{1}{c}+c\right) \cdot \frac{1}{4 ! \cdot 2 !}\right] \\
\{6,0, \theta\}=\frac{b l\{0,0, f\}}{\operatorname{Det}} \cdot \frac{1}{c^{3}} *\left[-\left(\frac{1}{c^{2}}-c^{2}\right) \cdot \frac{1}{4 !}\right] \\
\{4,2, \theta\}=\frac{b l\{0,0, f\}}{\operatorname{Det} \cdot \frac{1}{c} *\left[+\left(\frac{1}{c^{2}}-c^{2}\right) \cdot \frac{1}{4 !}\right]} \\
\{2,4, \theta\}=\frac{b l\{0,0, f\}}{\operatorname{Det}} \cdot c *\left[-\left(\frac{1}{c^{2}}-c^{2}\right) \cdot \frac{1}{4 !}\right] \\
\{0,6, \theta\}=\frac{b l\{0,0, f\}}{\operatorname{Det}} \cdot c^{3} *\left[+\left(\frac{1}{c^{2}}-c^{2}\right) \cdot \frac{1}{4 !}\right]
\end{gathered}
$$

The values of $\{m, n, \theta\} ; m+n<=6$ are tabulated in Appendix I. Attention is asked for the perfect symmetry in values for $c$ in relation to those of $1 / c$. 
An analytical solution is given in Reference 1, which in our variables reads

$$
\frac{\theta-\theta_{r}}{b l f}=-\frac{1-\xi^{2}}{2 c}+\frac{16}{\pi^{3} c} \cdot \sum_{n=0}^{\infty}(-1)^{n} \cdot \frac{\cos -[(2 n+1)(\pi \xi / 2)] \cdot \cosh -[(2 n+1)(\pi \eta c / 2)]}{(2 n+1)^{3} \cdot \cosh -[(2 n+1)(\pi c / 2)]}
$$

Since the symmetry in $\xi$ and $\eta$ is difficult to notice, comparison with our results is complicated; however, heat fluxes are also somewhat hidden. What we can do is calculate $\{0,0, \theta\}$ bysubstituting $\xi=0$ and $\eta=0$ for various values of $c$ and $n<=3$. This gives the following results:

$\begin{array}{cccc}c & \text { Our result } & \text { Exact solution } & \text { Discr. (\%) } \\ 1.00000 & -0.29167 & -0.29469 & -1.02 \\ 0.90909 & -0.29019 & -0.29315 & -1.01 \\ 0.82645 & -0.28581 & -0.28863 & -0.976 \\ 0.75131 & -0.27875 & -0.28132 & -0.912 \\ 0.68030 & -0.26930 & -0.27155 & -0.829 \\ 0.62092 & -0.25787 & -0.25974 & -0.718\end{array}$

so that our approximation, although only four terms were evaluated, is already accurate up to 1 per cent or better.

The temperature gradients as reported in Appendix I are given in terms of $\partial \theta / \partial \xi$ and $\partial \theta / \partial \eta$. Heat flux densities are calculated as

$$
q_{x}=-\lambda \cdot \frac{\partial \theta}{\partial x}=-\frac{\lambda}{l} \cdot \frac{\partial \theta}{\partial \xi} ; \quad q_{y}=-\lambda \cdot \frac{\partial \theta}{\partial y}=-\frac{\lambda}{l} \cdot \frac{\partial \theta}{\partial \eta}
$$

This ratios of the heat flux densitics at the centre of the edges are given as $q_{y} / q_{x}$ in Appendix I.

Obviously, even better approximations might be realized through calculation of higher derivatives. However, the development of the eleven $10 \times 10$ determinants into an exact formulation is looked upon as almost a Gordian knot. Numerical development is restricted, due to the poor condition of the determinants; the coefficients of the $4 \times 4$ determinants differ by three orders of magnitude, and those of the $10 \times 10$ determinants by as much as 15 . Therefore, the values are successively approximated, i.e. in step 1 a first approximation for, e.g., $\{0,0, \theta\}$ through $\{6,0, \theta\}$ is developed from the $4 \times 4$ determinants; in a second step, corrections of and first approximations for $\{0,0, \theta\}$ through $\{6,0, \theta\}$ and first approximations of $\{8,0, \theta\}$ through $\{10,0, \theta\}$ are calculated from the $6 \times 6$ determinants with, on the right-hand side, four zero and two non-zero, values, calculated from the first approximations of $\{0,0, \theta\}$ through $\{6,0, \theta\}$.

The procedure may be repeated as many times as desired, leading to the following result (Appendixes II and III):

$\begin{array}{cccc}c & \text { Our result } & \text { Exact solution } & \text { Discr. }(\%) \\ 1.00000 & -0.29457 & -0.29469 & -0.0407 \\ 0.90909 & -0.29304 & -0.29315 & -0.0375 \\ 0.82645 & -0.28853 & -0.28863 & -0.0346 \\ 0.75131 & -0.28125 & -0.28132 & -0.0249 \\ 0.68030 & -0.27152 & -0.27155 & -0.0110 \\ 0.62092 & -0.25974 & -0.25974 & -0.0000\end{array}$




\section{CONCLUSIONS}

The method described is not limited to two dimensions, nor does it require linearity of the differential equation. Dependent upon the effort that one wishes to spend, one may approximate the solution as closely as is desired.

\section{APPENDIX I}

Results of 4 terms approximation (prog 300-5)

$\mathrm{b} / 1: 0.62$

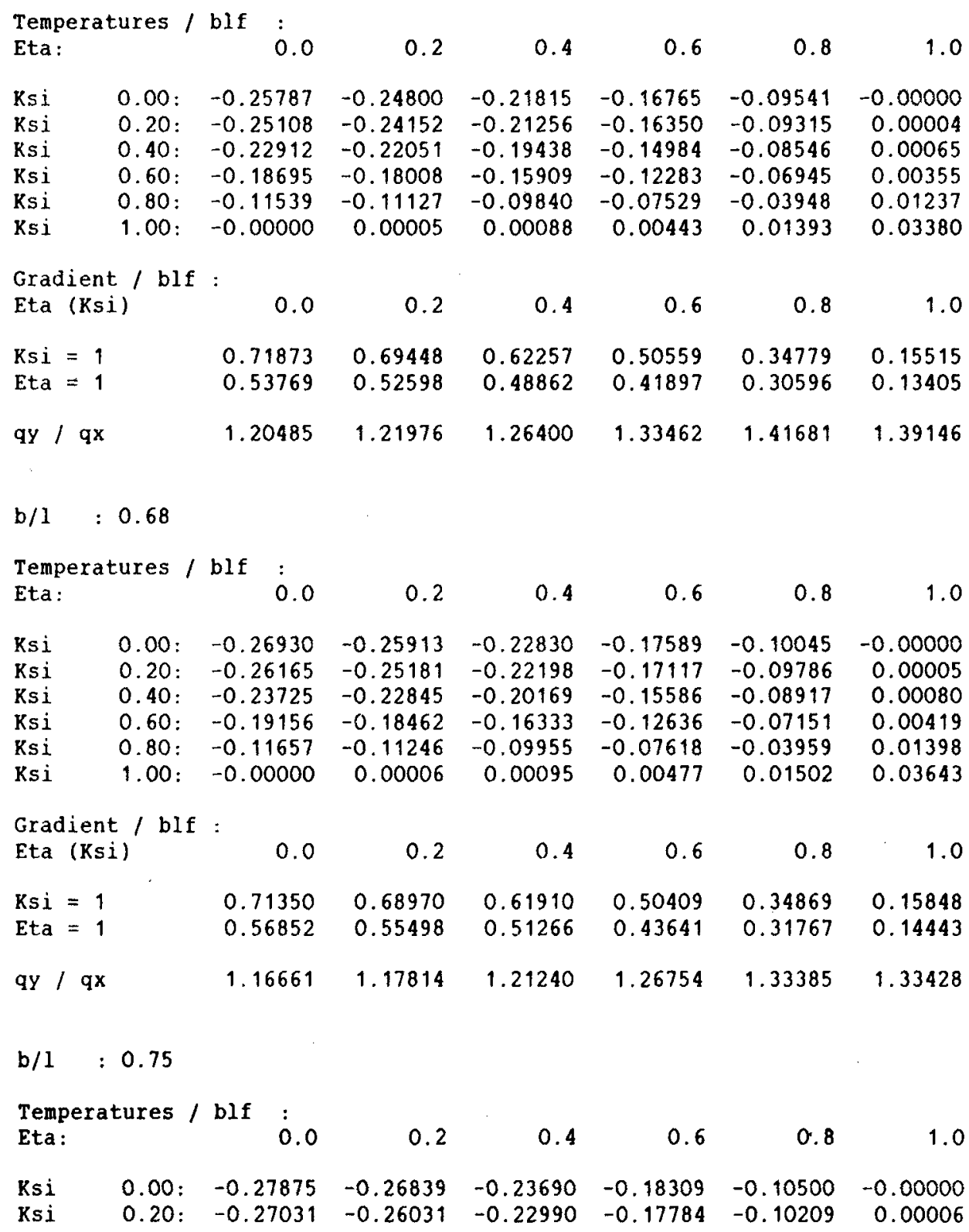




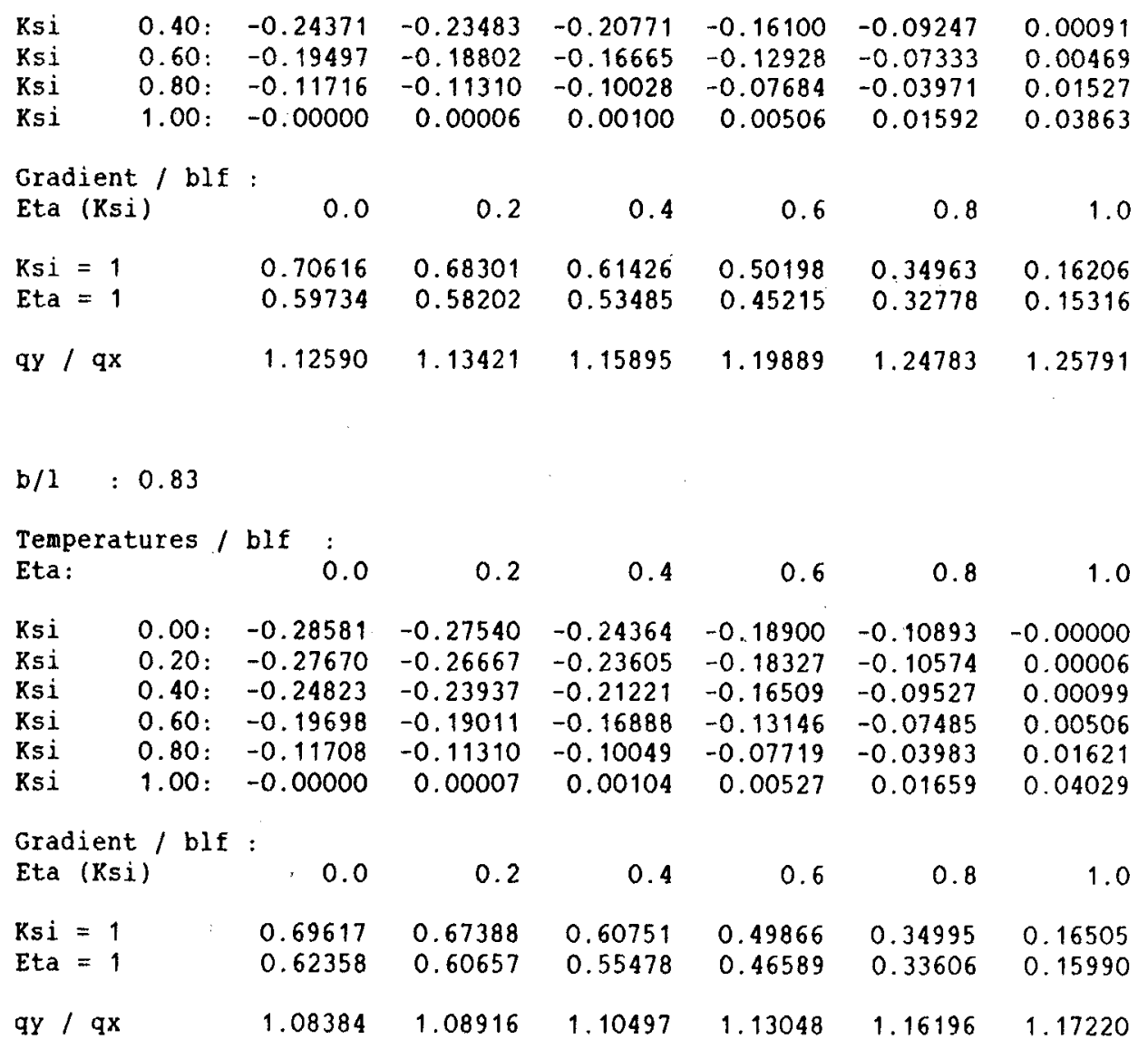

$\mathbf{b} / \mathbf{1}: 0.91$

Temperatures / blf :

$\begin{array}{llllllll}\text { Ksi } & 0.00: & -0.29019 & -0.27987 & -0.24827 & -0.19343 & -0.11215 & -0.00000 \\ \text { Ksi } & 0.20: & -0.28052 & -0.27060 & -0.24018 & -0.18730 & -0.10870 & 0.00006 \\ \text { Ksi } & 0.40: & -0.25057 & -0.24185 & -0.21499 & -0.16799 & -0.09751 & 0.00104 \\ \text { Ksi } & 0.60: & -0.19744 & -0.19073 & -0.16989 & -0.13281 & -0.07602 & 0.00529 \\ \text { Ksi } & 0.80: & -0.11624 & -0.11240 & -0.10012 & -0.07719 & -0.03991 & 0.01681 \\ \text { Ksi } & 1.00: & -0.00000 & 0.00007 & 0.00107 & 0.00539 & 0.01699 & 0.04132\end{array}$

Gradient / blf :

$\begin{array}{lllllll}\text { Eta (Ksi) } & 0.0 & 0.2 & 0.4 & 0.6 & 0.8 & 1.0\end{array}$

\begin{tabular}{|c|c|c|c|c|c|c|}
\hline $\mathrm{Ksi}=1$ & 0.68310 & 0.66185 & 0.59842 & 0.49368 & 0.34911 & 0.16677 \\
\hline Eta & 0.64679 & 0.62821 & 0.57211 & 0.47742 & 0.34236 & 0.16442 \\
\hline qy $/$ & 1.04154 & 1.04409 & 1.05165 & $\cdot 1.06378$ & 1.07875 & 1.08453 \\
\hline
\end{tabular}


$b / 1: 1.00$

Temperatures / blf :

Eta:

$\begin{array}{llllll}0.0 & 0.2 & 0.4 & 0.6 & 0.8 & 1.0\end{array}$

$0.00:-0.29167$

$-0.28160-0.25060$

$-0.19627$

$-0.11460$

$-0.00000$

Ksi $\quad 0.20:-0.28160$

$-0.27193-0.24213$

$-0.18980$

$-0.11093$

0.00007

Ksi $\quad 0.40:-0.25060$

$-0.24213-0.21593-0.16960$

$-0.09913$

0.00107

Ksi

$0.60:-0.19627$

$-0.18980$

$-0.16960$

$-0.13327$

$-0.07680$

0.00540

Ksi $\quad 0.80:-0.11460$

$-0.11093$

$-0.09913-0.07680$

$-0.03993$

0.01707

$\mathrm{Ksi}$

0.00007

$0.00107 \quad 0.00540$

0.01707

0.04167

Gradient / blf :

Eta (Ksi)

0.0

0.2

0.4

0.6

0.8

1.0

Ksi $=1$

0.66667

0.64667

0.58667

0.48667

0.34667

0.16667

Eta $=1$

0.66666

0.64666

0.58667

0.48667

0.34667

0.16667

$q y / q x$

1.00000

1.00000

1.00000

1.00001

1.00001

1.00001

$b / 1: 1.10$

Temperatures / blf :

Eta: $\quad 0.0$

$0.2 \quad 0.4$

0.6

0.8

1.0

$\mathrm{Ksi}$

$0.00: \quad-0.29019-0.28052$

$-0.25057-0.19744$

$-0.11624$

$-0.00000$

Ksi

$0.20:$

$-0.27987$

$-0.27060$

$-0.24185$

$-0.19073$

$-0.11240$

0.00007

Ksi

$0.40:-0.24827$

$-0.24018$

$-0.21499-0.16989$

$-0.10012$

0.00107

Ksi

$0.60:-0.19343-0.18730$

$-0.16799-0.13281$

$-0.07719$

0.00539

Ksi

$\begin{array}{ll}0.80: & -0.11215 \\ 1.00: & -0.00000\end{array}$

0.00006

$\begin{array}{rr}0.09751 & -0.07602 \\ 0.00104 & 0.00529\end{array}$

0.01681

0.04132

Gradient / blf :

Eta (Ksi)

0.0

0.2

0.4

0.6

0.8

1.0

$\mathrm{Ksi}=1$

0.64679

0.62821

0.57211

0.47742

0.34236

0.16442

Eta $=1$

0.68310

0.66185

0.59842

0.49368

0.34911

0.16677

qy / qx

0.96012

0.95778

0.95090

0.94005

0.92701

0.92207

$b / 1: 1.21$

Temperatures / blf :

Eta: $\quad 0.0$

0.2

0.4

0.6

0.8

1.0

Ksi

$0.00:-0.28581$

$-0.27670$

$-0.24823$

$-0.19698$

$-0.11708$

$-0.00000$

Ksi

$0.20: \quad-0.27540$

$-0.26667$

$-0.23937$

$-0.19011$

$-0.11310$

0.00007

$\mathrm{Ksi}$

$0.60:-0.18900$

$-0.23605$

$-0.21221$

$-0.10049$

0.00104

Ksi

$0.80:-0.10893$

$-0.18327$

$-0.07719$

0.00527

$K s i$

1.00: -0.00000

-0.10574
0.00006

$-0.09527$

$-0.13146$

$-0.03983$

0.01659

$0.00099 \quad 0.00506$

0.01621

0.04029

Gradient / blf :

Eta (Ksi)

0.0

0.2

0.4

0.6

0.8

1.0

Ks $i=1$

0.62359

0.60658

0.55478

$0: 46589$

0.33606

0.15990

Eta $=1$

0.69617

0.67387

0.60751

0.49866

0.34995

0. 16505

qy / qx

0.92265

0.91814

0.90501

0.88459

0.86062

0.85310 
$b / 1: 1.33$

Temperatures / bif :

Eta: $\quad 0.0$

0.2 .0 .4

0.6

0.8

1.0

Ksi

$0.00: \quad-0.27875-0.27031$

$-0.24371$

$-0.19497$

$-0.11716$

$-0.00000$

Ksi $\quad 0.20: \quad-0.26839-0.26032$

$-0.23483-0.18802$

$0.40:-0.23690$

$-0.22991$

$-0.20772$

$-0.16665$

$-0.11310$

$-0.17784$

$-0.16100-0.12928$

$-0.10028$

0.00006

Ksi $\quad 0.60: \quad-0.18309$

Ksi $\quad 0.80:-0.10500$

$-0.10209$

$-0.09247-0.07333$

$-0.07684$

0.00100

Ksi

1.00: -0.00000

0.00006

0.00091

0.00469

0.01527

0.00506

0.01592

0.03863

Gradient / blf :

Eta (Ksi)

0.0

0.2

0.4

0.6

0.8

1.0

$\mathrm{Ksi}=1$

0.59734

0.58203

0.53486

0.45215

0.32778

0.15316

Eta $=1$

0.70616

0.68301

0.61426

0.50198

0.34963

0.16206

qy / qx

0.88818

0.88167

$0.86285 \cdot 0.83411$

0.80140

0.79498

$\mathrm{b} / 1: 1.46$

Temperatures / blf :

Eta: $\quad 0.0$

0.2

$0: 4$

0.6

0.8

1.0

Xsi

$0.00:-0.26930$

$-0.26165$

$-0.23725$

$-0.19156$

$-0.11657$

$-0.00000$

Ksi $\quad 0.20:-0.25913$

$-0.25182$

$-0.22845$

$-0.18462$

$-0.11246$

0.00006

Ksi $\quad 0.60:-0.17590$

$-0.22198$

$-0.20169-0.16333$

$-0.09955$

0.00095

Ksi $\quad 0.80:-0.10045$

$-0.17117$

$-0.15586-0.12636$

$-0.07618$

0.00477

Ksi

$\begin{array}{ll}0.80: & -0.10045 \\ 1.00: & -0.00000\end{array}$

0.00005

$-0.08917$

$-0.07151$

$-0.03959$

0.01502

0.00080

0.00419 .

0.01398

0.03643

Gradient / bIf :

Eta (Ksi)

0.0

0.2

0.4

0.6

0.8

1.0

$\mathrm{Ksi}=1$

0.56852

0.55499

0.51267

0.43642

0.31767

0.14443

Eta $=1$

0.71349

0.68969

0.61909

0.50409

0.34869

0.15848

$q y / q x$

0.85719

0.84880

0.82481

0.78894

0.74971

0.74947

$b / 1: 1.61$

Temperatures / blf :

0.0

0.2

0.4

0.6

0.8

1.0

Ksi

$0.00:-0.25787$

$-0.25109$

$-0.22913$

$-0.18695$

$-0.11539$

$-0.00000$

$\mathrm{Ksi} \quad 0.20:-0.24800$

$\begin{array}{lll}-0.24152 & -0.22051 & -0.18008\end{array}$

$-0.11127$

0.00005

Ksi

$0.40:-0.21815$

$-0.21257$

$-0.19438$

$-0.15909$

$-0.09840$

0.00088

Ksi $\quad 0.80:-0.09541$

$-0.16350$

$-0.14984$

$-0.12283$

$-0.07529$

0.00443

$\mathrm{Ksi}$

1.00: $\quad-0.00000$

0.00004

0.00065

0.06945

$-0.03948$

0.01393

Gradient / blf :

Eta (Ksi)

0.0

0.2

0.4

0.6

0.8

0.03380

$\mathrm{K} s \mathrm{i}=1$

0.53769

Eta $=1$

0.71873

0.52598

0.48863

0.41898

0.30596

1.0

qy / qx

0.82998

0.81983

0.79114

0.50559

0.34779

0.13405

0.15516

0.74928

0.70582

0.71867 


\section{APPENDIX II}

Results of 10 terms approximation (prog 300 -6)

b/1 0.62

Temperatures / blf :

Eta :

0.0

0.2

0.4

0.6

0.8

1.0

$\begin{array}{llllllll}\text { Ksi } & 0.00: & -0.25974 & -0.24980 & -0.21974 & -0.16887 & -0.09608 & -0.00000 \\ \text { Ksi } & 0.20: & -0.25313 & -0.24351 & -0.21437 & -0.16495 & -0.09402 & -0.00000 \\ \text { Ksi } & 0.40: & -0.23159 & -0.22299 & -0.19684 & -0.15215 & -0.08726 & -0.00000 \\ \text { Ksi } & 0.60: & -0.18972 & -0.18302 & -0.16250 & -0.12688 & -0.07386 & -0.00007 \\ \text { Ksi } & 0.80: & -0.11756 & -0.11380 & -0.10214 & -0.08140 & -0.04907 & -0.00034 \\ \text { Ksi } & 1.00: & -0.00000 & 0.00002 & 0.00024 & 0.00074 & 0.00146 & 0.00484 \\ \text { Gradient / blf : } & & & & & & \\ \text { Ksi = 1: } & & 0.73221 & 0.71280 & 0.65469 & 0.55088 & 0.37496 & 0.08741 \\ \text { Eta = 1 : } & & 0.54119 & 0.53069 & 0.49632 & 0.42765 & 0.29660 & 0.03690 \\ \text { qY / qx : } & & 1.19035 & 1.19906 & 1.22091 & 1.25025 & 1.27394 & 0.67983\end{array}$

$\mathrm{b} / 10.68$

Temperatures / blf :

Eta :

0.0

0.2

0.4

0.6

0.8

1.0

Ksi

0.00 :

$-0.27152$

$-0.26127$

$-0.23021$

$-0.17738$

$-0.10128$

$-0.00000$

Ksi

0.20 :

$-0.25412$

$-0.22411$

$-0.17293$

$-0.09893$

$-0.00000$

Ksi

0.40

$-0.23993$

$-0.20445$

Ksi

0.60 :

$-0.18764$

$-0.16694$

$-0.15853$

$-0.09131$

$-0.13079-0.07650$

$-0.00000$

$-0.10332$

$-0.08257$

$-0.04991$

$-0.00003$

1. 00 :

$-0.11866$

-0.11492
0.00001

0.00013

0.00039

0.00099

0.00006

0.00524

Gradient / blf :

$0.72609 \quad 0.70682$

0.64795

0.54079

0.36049

0.07370

Eta $=1$ :

0.57290

0.56092

0.52210

0.44615

0.30657

0.04561

$\mathrm{qy} / \mathrm{qx}$ :

1. 15521

1. 16189

1.17973

$1.20790 \quad 1.24509$

0.90612

b/1 0.75

Temperatures / blf :

Eta :

0.0

0.2

0.4

0.6

0.8

1. 0

Ksi

$0.00: \quad-0.28125 \quad-0.27082$

$-0.23911$

$-0.18484$

$-0.10600$

$-0.00000$

$\mathrm{K} s \mathrm{i}$

$0.20:-0.27292-0.26289$

$-0.23232$

$-0.17987$

$-0.10337$

$-0.00000$

Ksi

$0.40:-0.24654-0.23771$

$-0.21079$

$-0.16398$

$-0.09493$

$-0.00000$

KsI

0.60 :

$-0.19777$

$-0.19106$

$-0.17037$

$-0.13400$

$-0.07882$

0.00000

$\mathrm{Ksi}$

$1.00:-0.00000$

0.00001

0.00007

-0.08339
0.00019

0.05060

0.00036

0.00582

Gradient / blf :

$\mathrm{Ksi}=1$ :

$\begin{array}{ll}0.71774 & 0.69877\end{array}$

0.64005

0.53214

0.35182

0.06839

Eta $=1$ :

0.60265

0.58924

0.54618

0.46336

0.31549

0.05210

$q y / q x$ :

1.11759

1.12238

1.13581

1.15896

1.19355

1.01392 
b/1 0.83

Temperatures / blf :

Eta :

0.0

0.2

0.4

0.6

0.8

1.0

Ks i

$0.00: \quad-0.28853$

$-0.27806$

$-0.24610$

$-0.19100$

$-0.11010$

$-0.00000$

Ksi

0.20 :

$-0.27948$

$-0.26943$

$-0.23870$

$-0.18556$

$-0.10721$

$-0.00000$

Ksi

0.60 :

$-0.25112$

-0.23870
-0.24234

$-0.16834$

$-0.09803$

$-0.17263$

$-0.13639$

$-0.08074$

0.00000

Ks i

0.80 :

$-0.11891$

$-0.11531$

$-0.10410$

$-0.08380$

$-0.05112$

0.00003

0.00000

0.00003

0.00010

0.00057

0.00628

Gradient / blf :

$\mathrm{Ksi}=1:$

0.70667

0.68818

0.63046

0.52385

0.00082

0.00628

Eta $=1:$

0.62986

0.61513

0.56815

0.47898

0.34612

0.06600

$q y / q x$ :

1.07849

1.08155

1.09041

1. 1063

0.32340

0.05630

1.03217

$b / 10.91$

Temperatures / blf :

Eta :

0.0

0.2

0.4

0.6

$0.8 \quad 1.0$

Ks i

$0.00: \quad-0.29304$

$\begin{array}{ll}-0.28269 & -0.25093\end{array}$

$-0.19566$

Ksi

$0.20:-0.28340$

$-0.27349-0.24301$

$-0.18981$

-0.11349
-0.11038
-0.10053

$-0.00000$

Ksi

$0.40: \quad-0.25346$

$-0.24484$

$-0.21825$

$-0.17144$

$-0.10053$

$-0.00000$

Ksi

0.60 :

$-0.20004$

$-0.19360$

$-0.17360$

$-0.13786$

$-0.08222$

0.00000

$\mathrm{Ksi}$

$1.00:$

$-0.00000$

0.00000

$-0.08375-0.05145$

0.00004

0.00072

Gradient / blf :

$\mathrm{Ksi}=1$ :

0.69250

0.67468

0.00001

0.00006

0.00083

0.00653

Eta $=1$ :

0.65408

0.61873

0.51494

0.34132

0.06386

$q y / q x$ :

1.03896

1.04043

1.04477

$1.05272 \quad 1.06455$

1.01861

b/1 1.00

Temperatures / blf :

Eta :

0.0

0.2

0.4

0.6

0.8

1.0

$\mathrm{Ksi}$

$0.00: \quad-0.29457 \quad-0.28449$

$-0.25340$

$-0.19869$

$-0.11611$

$-0.00000$

$\mathrm{K} 5 \mathrm{i}$

$0.20:-0.28449$

$-0.27486$

$-0.24507$

$-0.19251$

$-0.11280$

$-0.00000$

Ksi

$0.40:-0.25340-0.24507$

$-0.21923$

$-0.17321-0.10239$

0.00000

Ksi

$0.60:-0.19869$

$-0.19251$

$-0.17321$

$-0.13836$

$-0.08323$

0.00005

0.80

0.11611

$-0.11280$

0.10239

$-0.08323-0.05156$

0.00080

Ksi

$1.00:$

Gradient / blf :

$\mathrm{Ksi}=1$ :

0.67500

0.65803

0.00000

0.00005

0.00080

0.00660

Eta $=1$ :

0.67500

0.65803

0.60451

0.50471

0.33618

0.06144

$q y / q x$ :

1.00000

1.00000

1.00000

1.00000

1.00000

0.99999 
b/1 1.10

Temperatures / blf :

Eta :

0.0

0.2

0.4

0.6

0.8

1.0

Ksi

Ksi

Ks i

0.00 :

0.20 :

0.40 :

0.60 :

0.80 :

1. 00 :

$-0.29304$

$-0.28340$

$-0.27349$

$-0.24301$

$-0.18981$

$\mathrm{Ksi}$

$\mathrm{Ksi}$

bIf :

Gradient / blf :

$\mathrm{Ks} \mathbf{i}=1$ :

Eta $=1$ :

0.65408

0.69251

0.63814

0.67466

0.96250

0.96112

qy / qx :

0.58763

0.61863

0.49275

0.51470

0.95704

0.94960

0.06316

b/1 1.21

Temperatures / blf :

Eta :

0.0

0.2

0.4

0.6
$0.00:$

0.20 :

0.40 :

0.60 :

0.80 :

$1.00:$

$-0.28853$

$-0.27948$

$-0.25112$

$-0.24234$

Ksi

$-0.27806$

$-0.24610$

$-0.19100$

$-0.26943$

$-0.23870$

$-0.18556$

$-0.10721$

Ks i

$-0.11010$

$-0.00000$

$-0.00000$

Gradient / blf :

\section{Ks $i=1$.}

Eta $=1$ :

0.62986

0.70669

0.61512

0.68811

0.92726

0.92451

0.56812
0.63009

0.47892

0.52297

0.91659

0.90246
0.33020

0. 34088

0.05887

0.8

0.97527

0.32328

0.34455

0.88082

0.00000

$-0.00000$

0.00000

0.00005

0.00080

0.00648

0.93849

$-0.11891$

$-0.11531$

$-0.10410$

$-0.08380$

$-0.05112$

0.00056 $-0.00000$ 0.00000 0.00004 0.00072 0.00611

0.05581

0.06350

0.94025

b/1 1.33

Temperatures / blf :

Eta :

0.0

0.2

0.4

0.6

0.8

1.0

$\begin{array}{lll}\text { Ksi } & 0.00: & -0.28125 \\ \text { Ksi } & 0.20: & -0.27082 \\ \text { Ksi } & 0.40: & -0.23911 \\ \text { Ksi } & 0.60: & -0.18484 \\ \text { Ksi } & 0.80: & -0.10600 \\ \text { Ksi } & 1.00: & -0.00000\end{array}$

$-0.27292$

$-0.24654$

$-0.19777$

$-0.11913$

0.00000

$\begin{array}{ll}-0.26289 & -0.23771\end{array}$

$-0.21071$

$-0.19106$

$-0.17037$

$-0.11545$

$-0.10400$

$-0.00001$

$-0.17987$

$-0.16398$

$-0.13400$

$-0.08339$

0.00001

$-0.10337$

$-0.09493$

$-0.07882$

$-0.05061$

0.00005

$-0.00000-0.00000$

0.00034

0.00058

Gradient / blf :

$\mathrm{Ksi}=1$ :

Eta $=1$ :

0.602 .65

0.58924

0.54617

0.46331

0.31539

0.00541

$q Y$ / qX :

0.71782

0.69859

0.63909

0.52987

0. 34772

0.05116

0.89489

0.8907 .5

0.87914

0.85925

0.82834

0.90941 
b/1 1.46

\begin{tabular}{|c|c|c|c|c|c|c|c|}
\hline \multicolumn{2}{|c|}{ Eta : } & 0.0 & 0.2 & 0.4 & 0.6 & 0.8 & 1.0 \\
\hline $\mathrm{Ksi}$ & $0.00:$ & -0.27152 & -0.26401 & -0.23993 & -0.19438 & -0.11866 & 0.00001 \\
\hline $\mathrm{Ksi}$ & $0.20:$ & -0.26127 & -0.25412 & -0.23116 & -0.18764 & -0.11492 & -0.00001 \\
\hline $\mathrm{Ksi}$ & $0.40:$ & -0.23021 & -0.22411 & -0.20445 & -0.16694 & -0.10332 & 0.00000 \\
\hline $\mathrm{Ksi}$ & $0.60:$ & -0.17738 & -0.17293 & -0.15853 & -0.13079 & -0.08258 & 0.00007 \\
\hline$K s i$ & $0.80:$ & -0.10128 & -0.09893 & -0.09131 & -0.07650 & -0.04992 & 0.00042 \\
\hline Ksi & 1.00: & -0.00000 & -0.00000 & -0.00000 & -0.00003 & 0.00003 & 0.00434 \\
\hline \multicolumn{8}{|c|}{ Gradient / blf : } \\
\hline $\mathrm{Ksi}=1:$ & & 0.57290 & 0.56092 & 0.52209 & 0.44613 & 0.30647 & 0.04373 \\
\hline Eta $=1:$ & & 0.72632 & 0.70647 & 0.64586 & 0.53580 & 0.35144 & 0.05942 \\
\hline$q y / q x$ : & & 0.86592 & 0.86025 & 0.84493 & 0.82029 & 0.78324 & 0.92810 \\
\hline
\end{tabular}

b/1 1.61

Temperatures / blf :

$\begin{array}{lllllll}\text { Eta: } & 0.0 & 0.2 & 0.4 & 0.6 & 0.8 & 1.0\end{array}$

$\begin{array}{lllllllr}\text { Ksi } & 0.00: & -0.25974 & -0.25313 & -0.23159 & -0.18972 & -0.11756 & 0.00003 \\ \text { Ksi } & 0.20: & -0.24980 & -0.24351 & -0.22299 & -0.18302 & -0.11380 & -0.00002 \\ \text { Ksi } & 0.40: & -0.21974 & -0.21437 & -0.19684 & -0.16250 & -0.10215 & -0.00002 \\ \text { Ksi } & 0.60: & -0.16887 & -0.16495 & -0.15215 & -0.12688 & -0.08142 & 0.00012 \\ \text { Ksi } & 0.80: & -0.09608 & -0.09402 & -0.08726 & -0.07386 & -0.04910 & 0.00033 \\ \text { Ksi } & 1.00: & -0.00000 & -0.00000 & -0.00000 & -0.00007 & -0.00039 & 0.00306\end{array}$

Gradient / blf :

$\begin{array}{lllllll}\text { Ksi = 1: } & 0.54119 & 0.53069 & 0.49631 & 0.42764 & 0.29648 & 0.03325 \\ \text { Eta = 1: } & 0.73276 & 0.71219 & 0.65062 & 0.54103 & 0.35702 & 0.05906 \\ \text { qY / qX : } & 0.84072 & 0.83328 & 0.81397 & 0.78556 & 0.74772 & 1.10297\end{array}$

\section{APPENDIX III}

Results of exact formula (prog 300-10)

Prog. Calslaw, versie 840802

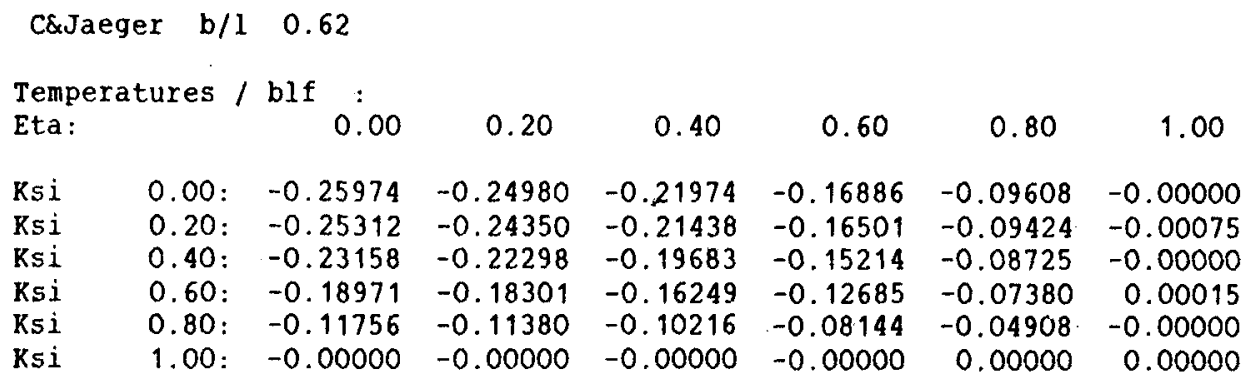




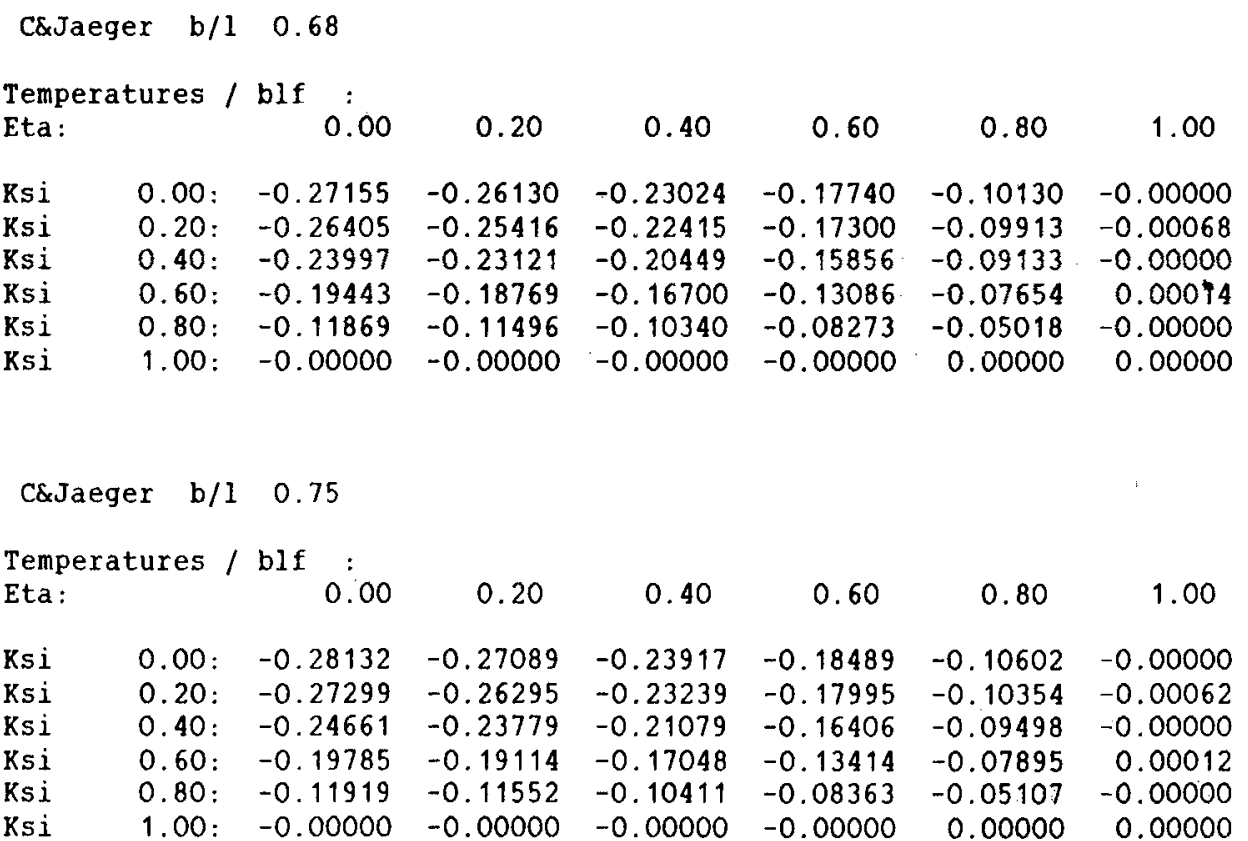

C\&Jaeger b/l 0.83

Temperatures / blf

Eta:

0.00

$\begin{array}{llllll}\text { Ksi } & 0.00: & -0.28863 & -0.27816 & -0.24619 & -0 \\ \text { Ksi } & 0.20: & -0.27958 & -0.26953 & -0.23879 & -0 \\ \text { Ksi } & 0.40: & -0.25122 & -0.24244 & -0.21548 & -0 \\ \text { Ksi } & 0.60: & -0.19980 & -0.19320 & -0.17277 & -0 \\ \text { Ksi } & 0.80: & -0.11897 & -0.11539 & -0.10423 & -0 . \\ \text { Ksi } & 1.00: & -0.00000 & -0.00000 & -0.00000 & -0\end{array}$

$0.20 \quad 0.40 ; 0.60$

0.80

1.00

$-0.19106-0.11014-0.00000$

$-0.18565-0.10737-0.00056$

$\begin{array}{lll}-0.16845 & -0.09811 & -0.00000\end{array}$

$\begin{array}{lll}-0.13658 & -0.08094 & 0.00011\end{array}$

$\begin{array}{lll}-0.08408 & -0.05174 & -0.00000\end{array}$

$\begin{array}{lll}-0.00000 & -0.00000 & 0.00000\end{array}$

c\&Jaegex $\quad b / 1 \quad 0.91$

Temperatures / blf :

Eta: $\quad 0.00$

$\begin{array}{lll}0.20 & 0.40 \quad 0.60\end{array}$

0.80

1.00

$\begin{array}{lllllllr}\text { Ksi } & 0.00: & -0.29315 & -0.28280 & -0.25103 & -0.19575 & -0.11354 & -0.00000 \\ \text { Ksi } & 0.20: & -0.28352 & -0.27360 & -0.24312 & -0.18992 & -0.11053 & -0.00051 \\ \text { Ksi } & 0.40: & -0.25357 & -0.24496 & -0.21838 & -0.17158 & -0.10064 & -0.00000 \\ \text { Ksi } & 0.60: & -0.20014 & -0.19371 & -0.17376 & -0.13808 & -0.08248 & 0.00010 \\ \text { Ksi } & 0.80: & -0.11798 & -0.11452 & -0.10371 & -0.08406 & -0.05215 & -0.00000 \\ \text { Ksi } & 1.00: & -0.00000 & -0.00000 & -0.00000 & -0.00000 & -0.00000 & 0.00000\end{array}$ 


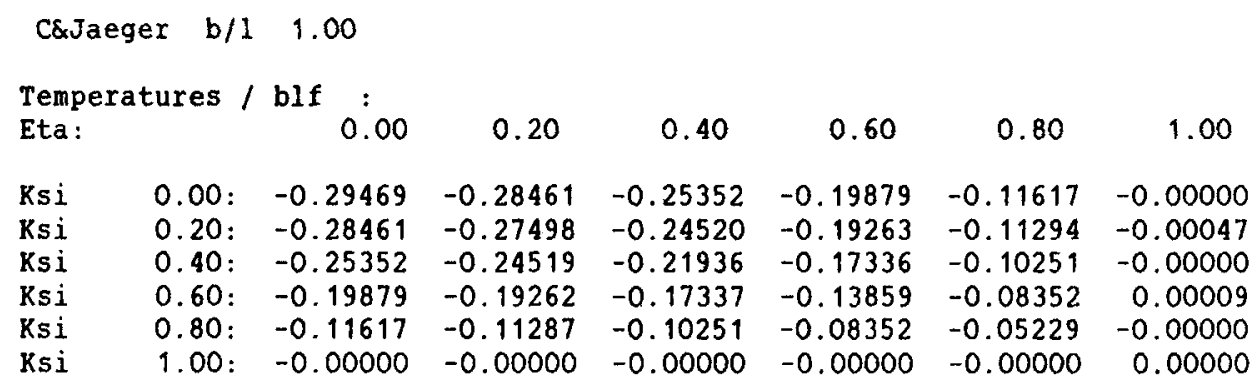

\section{APPENDIX IV: NOTATION}

$\begin{array}{llll}\theta & =\text { Temperature } & \text { Units } & \text { Dimension } \\ p & {[\mathrm{~K}]} & {[\theta]} \\ x, y, z & =\text { Coordinates } & & \\ \xi, \eta & =\text { Dimensionless coordinates } & {[\mathrm{m}]} & {[L]} \\ l & =\text { Length of rectangle } & {[\mathrm{m}]} & \\ b & =\text { Width of rectangle } & {[\mathrm{m}]} & {[L]} \\ f & =\text { Function } & & {[L]} \\ q & =\text { Heat generated per unit volume } & {\left[\mathrm{W} \cdot \mathrm{m}^{-3}\right]} & \\ \lambda & =\text { Heat conductivity } & {\left[\mathrm{W} \cdot \mathrm{m}^{-1} \cdot \mathrm{K}^{-1}\right]} & {\left[M \cdot L^{-1} \cdot T^{-3}\right]} \\ & & & {\left[M \cdot L^{+1} \cdot T^{-3} \cdot \theta^{-1}\right]}\end{array}$

General:

$$
\begin{aligned}
\theta_{[x, y, z]} & =\text { value of } \theta \text { at } x, y, z \\
\left\{p_{x}, p_{y}, p_{z}, \theta\right\} & =\frac{\partial^{p_{x}+p_{y}+p_{z}}}{\partial x^{p_{x}} \cdot \partial y^{p_{y}} \cdot \partial z^{p_{z}}} \theta_{[x, y, z]}
\end{aligned}
$$

\section{REFERENCES}

1. H. S. Carlslaw and J. C. Jaeger, Conduction of Heat in Solids, 2nd edn, Clarendon Press, Oxford, 1976, p. 171.

2. R. D. Small, 'Numerical methods for partial differential equations based on power series', Int.j. numer. methods eng., 19, 739-755 (1983). 